\title{
Can 'Openness-to-change' and 'Conservation' Values Predict the Diffusion of the Internet into European Homes?
}

\author{
Morten Blekesaune \\ Department of Sociology and Social Work, University of Agder, Kristiansand, \\ Norway \\ morten.blekesaune@uia.no
}

\begin{abstract}
This study investigated whether openness-to-change values (self-direction and stimulation) and conservation values (security and tradition) could predict the diffusion of the internet into homes by 2014, at the level of 159 European regions. All four value scales were correlated with the diffusion of the internet, after controlling for regional wealth and population density. Self-direction was by far the most consistent predictor of the diffusion of internet connections; a combination of self-direction and stimulation values could predict home internet use better than other combinations of human values. A longitudinal analysis, at the level of 25 countries, investigated the possibility of reverse causation (could the internet affect human values?), between 2004 and 2014 . The study found no such effects.
\end{abstract}

\section{Keywords}

diffusion processes - European regions - digital divide - human values - internet

\section{Introduction}

The internet has expanded enormously over the past two decades. It has become more useful with the advent of high-speed access. Fast internet connections have entered private homes and are increasingly changing domestic life regarding social interactions and consumer behaviors (Takieddine and Sun 2015; Zhao 2006). However, the spread of the internet has been uneven, 
with vast differences among different countries and regions and among different age and gender groups (Guillen and Suarez 2005; Korupp and Szydlik 2005).

Previous studies have investigated the characteristics of leaders, laggers and non-adopters in the diffusion of the internet (e.g., Dauvin and Grzybowski 2014; Erumban and de Jong 2006; Wu et al. 2011). Some of these studies have also investigated the role of human values in the adoption of the internet (e.g., Bagchi et al. 2004; Erumban and De Jong 2006). Findings are inconsistent, however, certainly at aggregated levels (i.e., countries), where they have not been robust for other factors, such as economic wealth.

One possible explanation for the lack of robust and consistent findings is the use of value scales that were too general (i.e., Hofstede taxonomy) or from comparing geographical units that were too heterogeneous (i.e., countries). In contrast to previous studies, the present study investigated whether opennessto-change and conservation values in the Schwartz taxonomy, retrieved from the European Social Survey (ESS), could predict diffusion of the internet into homes, retrieved from the Community Statistics on Information Society (CSIS), at the level of ${ }_{159}$ European regions. First, we will consider the theoretical links between human values and the diffusion of the internet.

\section{$2 \quad$ Background}

The notion that cultural factors, including human values, might explain the diffusion of innovative ideas and collaborations has a long tradition. In sociology, Max Weber (1934) presented some classical examples, most famously in the argument that modern capitalism evolved in Protestant (particularly Calvinist) regions of Europe. However, this type of explanation is not without problems. One issue relates to causal order: what came first, the cultural diffusion of values or the diffusion of innovative practices?

Another issue is the more general relationship between values and behavior. The sociology of culture has questioned the role of human values in explaining behavior altogether (e.g., Lizardo 2017). Swidler (1986) argued that 'culture' is merely a 'tool-kit' that people draw on to accomplish certain strategies of action. She presented this 'tool-kit' theory as an alternative to a model claiming that culture supplies values that affect people's behavior, a model she believed was flawed. Other sociologists have presented similar arguments, including Boltanski and Thévenot (1999) theory about moral justifications (Vaisey 2009).

Both debates come down to questions about how stable human values are, as related to more visible and material aspects of social change (Weber 1934), 
or related to actual behavior (Lizardo 2017; Swidler 1986). Theory cannot resolve these issues of temporal order and stability. The development of value scales and access to longitudinal data, at the levels of individuals and societies, may allow us to resolve some of these issues.

\subsection{General Human Values}

Value theory distinguishes between general human values (i.e., motivational constructs) and concrete values (i.e., how people value certain things) (Rokeach 1973; Schwartz 1992). The general human values (motivational constructs) guide people in evaluating and selecting certain objects (e.g., broadband connections). These values are abstract goals that transcend specific actions and situations (e.g., technical innovations). Consequently, they might serve as potential explanations for the diffusion of innovative ideas and practices. In contrast, concrete values (how people value certain things; e.g., broadband connections) might better be labeled preferences or attitudes, and causal relations with actual behavior is most likely reciprocal.

General human values can also change, but they change slowly, certainly during an adult life. In contrast, preferences and attitudes can change more abruptly (Hofstede 2001; Rokeach 1973). Human values might change, for example, after major events have occurred at young age (Daniel et al. 2013; Vecchione et al. 2016). Longitudinal data indicate that an increase in the importance of one value is typically accompanied by an increase in the importance of compatible values and a decrease in the importance of conflicting values (Bardi et al. 2009).

Some value taxonomies are available in large scale and comparative data including those developed by Hofstede $(1980 ; 1998 ; 2001)$, Inglehart (1990), and Schwartz (1992; 2012). The Schwartz taxonomy allows for investigations of general human values at more detailed dimensions compared to the other two taxonomies. The Hofstede (2001) taxonomy has been frequently used in business management research, including some research on the diffusion of information technologies (Bagchi et al. 2004; Erumban and De Jong 2006; Takieddine and Sun 2015), perhaps because it was originally developed for management purposes (Hofstede 1980).

The Schwartz $(1992 ; 2012)$ taxonomy was developed to identify universal values that are recognized in all major human cultures, albeit to varying degrees. The empirical analysis identified 10 general human values, which reflected a set of four larger, more general groups of values, known as openness-to-change, conservation, self-enhancement, and self-transcendence values. Schwartz (2012) argued that these values operated at the levels of individuals, institutions, and entire societies. 


\subsection{Why Should Values Affect the Diffusion of the Internet?}

Values can affect the diffusion of the internet, because values affect how we relate to a changing world. Individuals and societies holding openness-tochange values tend to welcome any change and encourage the pursuit of new ideas and experiences. In contrast, conservation values emphasize maintaining the status quo and avoiding threat (Piurko et al. 2011). Because the internet represents a major change in modern lives and societies, with unforeseen consequences, it is easy to hypothesize that individuals and societies that hold openness-to-change values are more likely to adopt this new development than individuals and societies that hold conservation values.

Openness-to-change values have two sub-dimensions in the Schwartz taxonomy: self-direction and stimulation. Self-direction values are based on making one's own decisions, instead of being told what to do by others. Individuals and societies that hold these values are likely to be particularly enthusiastic about the internet, primarily because internet-based information sources are difficult to control by governments and other authorities. Buchner (1988) presented a similar argument regarding the diffusion of telephones versus televisions. Authoritarian countries tended to hamper the diffusion of telephones, but not the diffusion of televisions, because televised materials were easier for governments to control. Similar arguments have also been made for the internet, which could be more difficult to control than telephones (Crenshaw and Robison 2006; Guillen and Suarez 2005).

Stimulation values are held by individuals and societies that search for excitement and new experiences. Because the internet is a potential source or mediator of excitement and experiences, individuals and societies that hold stimulation values are more likely to adopt internet connections and use the internet at home compared those that do not hold stimulation values.

The conservation values might also explain the diffusion of the internet. Conservation values include two sub-dimensions in the Schwartz taxonomy: security and tradition. Security values are held by individuals and societies that are concerned about social order and personal protection. Because individuals and societies that hold these values might see the internet as a potential threat to social order, they are less likely to adopt this new development than individuals and societies that do not hold security values. Tradition values are held by individuals and societies that accept the customs and ideas provided by traditional cultures. Because the internet might challenge traditional customs, individuals and societies that hold tradition values might refrain from adopting this new communication technology. 


\subsection{Why are Regions Important?}

Regions could be important for the diffusion of the internet, because the development of the internet depends on the preferences of many residents, and because the use and the utility of the internet affects all residents. Broadband connections are expensive; thus, they are financially sustainable only when a certain proportion of people living in a region are willing to pay for access to the internet. Additionally, the utility of accessing the internet at home depends on how much peers, family, and friends are also using the internet, and on whether local businesses and public services have established websites on the internet (DiMaggio and Garip 2011). This theory of positive externalties for adopting new technologies was originally developed for telephones (see Fisher 1992). Essentially, there was little reason to buy a telephone if friends and customers did not use them. DiMaggio and Garip (2011: 1892) argued that this explanation applied to the utility of many communication technologies, including telephones, e-mail, instant messaging, and social media, like Facebook and Twitter. Finally, the use of the internet also requires socialization and the development of skills in using the computer (Bobkowski and Smith 2013; van Deursen and van Dijk 2011).

Previous research has shown that internet access and participation depend on the characteristics of both individuals and the regions (Guillen and Suarez 2005). It is not simple to determine the most appropriate geographical level for investigating the roles of positive externalties and other contextual factors related to internet use at home. To some individuals, social networks and services are related to a city or a local community; in turn, other individuals might have networks that require communication over long distances or beyond national borders.

For the present study, we hypothesized that different European regions varied in both their general human values and the positive externalties involved in connecting to the internet. We investigated the first hypothesis about human values empirically, by splitting variance statistics between and within regions (an issue of internal validity). We investigated the second hypothesis about positive externalities by correlating human values to the diffusion of internet connection and internet use at the level of European regions (an issue of external validity and predictive power).

\subsection{Previous Findings}

The issue of how cultural factors might affect the diffusion of internet connection and internet use has been investigated previously at several levels, including individuals, households, regions, and nations. Some individual-level 
studies have investigated the roles of human values, including those described in the Schwartz taxonomy (Bagchi et al. 2015; Wu et al. 2011). Findings have indicated that openness-to-change values were associated with internet use (Bagchi et al. 2015) and online shopping (Wu et al. 2011); in contrast, conservation values were associated with less internet use (Bagchi et al. 2015).

Only a few studies have investigated internet use at the regional level, and we could not identify any studies that investigated the role of human values. A study conducted in the 50 states in the US showed that internet connections and internet use were associated with urban populations, education levels, and Putnam's social capital index (e.g., voting, trust, voluntarism) (Pick et al. 2015). A study conducted in 164 European (NUTS) regions showed that internet connections and internet use at home were associated with regional wealth, education levels, and younger populations (Vicente and López 2011).

More aggregate level studies have compared internet connections and internet use at the country level. Several studies have shown that the development and use of the internet were related to the economic wealth of a country (Bagchi et al. 2004; Crenshaw and Robison 2006; Erumban and De Jong 2006; Guillen and Suarez 2005). Some studies have also shown that telecommunication policies (i.e., deregulation) might facilitate the diffusion of the internet (Dauvin and Grzybowski 2014; Guillen and Suarez 2005).

Some country-level studies have investigated the Hofstede values (Bagchi et al. 2004; Erumban and De Jong 2006). Those findings indicated that the relevant values were also correlated with the economic wealth of the countries. Erumban and De Jong (2006) found that country level differences in expenditures on information and communication technologies were related to two of five values - power distance and uncertainty avoidance. However, they controlled for national wealth by using only a single dummy variable. Bagchi et al. (2004) found that only one of the four Hofstede values - individualism - was related to the adoption of six information technologies, after controlling for national wealth, linguistic variations, and income distributions.

Guillen and Suarez (2005) found that expenditure on tourism abroad was correlated with internet use, after controlling for economic and political factors. The authors interpreted the finding as support for a cultural explanation of the diffusion of the internet. Tourism abroad was taken as a proxy for 'culture of cosmopolitanism', an ideal type developed by Merton (1968), described as individuals with diverse social contacts that like to travel, are likely to move residence, and that pay attention to media about foreign and domestic affairs and the arts.

It is possible to link the findings above to the Schwartz taxonomy. For example, tourism abroad is also an indicator of openness-to-experience, and individualism is associated with the sub-dimension, self-direction. 
The country-level studies mentioned above were aggregate-level analyses that, in some cases, controlled for the demographic composition of the countries (e.g., the proportion of individuals over 65 years of age). However, those methods carry the risk of ecological fallacy, because age composition is also related to other modernization processes, including (first and second) demographic transitions (e.g., Lesthaeghe 2014). Age composition might also reflect previous migration, certainly at the regional level (Hadler 2006). Some of these fallacies can be avoided by using multilevel data or by standardizing variables. The present study used both these methods.

\section{Methods}

\subsection{A Three Stage Analysis}

This study tested the hypothesis that the diffusion of the internet could be explained by human values. First, we investigated variations in openness-tochange and conservation values among 159 European (largely NUT S2) regions. Second, we investigated whether geographical variations in human values might predict the diffusion of the internet among the 159 regions, by 2014. We selected the lowest geographical level possible for conducting an aggregate-level analysis. The year 2014 was the first year that broadband connections and internet use could be investigated in this number of regions. The main analysis rested on one crucial assumption: that the diffusion of the internet did not affect general human values. Our final analysis investigated this assumption longitudinally, at the level of 25 European countries over a 10-year period (2004-14).

\subsection{Dependent Variables: Internet Connections and Use}

The outcome variables were broadband connections at home and daily internet use. These data were collected from the "household" part of the csis $(\mathrm{N}=359,905)$. We examined the proportions of individuals, for internet use analyses, and the proportions of households, for internet access analyses. Eurostat did not allow individual researchers to access micro data with regional codes, but it had published the rates of internet connections and internet use at the regional (NUTS2) level. Internet connections and use varied by age and gender. Hence, we adjusted the regional aggregates for internet connections and internet use for the demographic composition of the regions, based on the indirect method of standardization. We estimated 12 age - and genderspecific rates of internet access and internet use in CSIs (2014) and we adjusted the rates of access and use with similar demographic data on age and gender groups from Eurostat (by January 2014). 


\subsection{Independent Variables}

Human values were investigated in the Ess, rounds $5^{-7}$, which held data on 151,573 respondents that lived in 159 regions and 27 countries. The Schwartz (2003) taxonomy in the Ess presented 21 portraits of individuals. Respondents were asked to 'think about how much each portrait is or is not like you', and to choose one of six possible responses, which ranged from 'very much like me' to 'not like me at all'. The four human value scales investigated comprised two items each, as follows:

Self-direction: (1) Thinking up new ideas and being creative is important to him. He likes to do things in his own original way. (2) It is important to him to make his own decisions about what he does. He likes to be free to plan and to choose his activities for himself.

Stimulation: (1) He likes surprises and is always looking for new things to do. He thinks it is important to do lots of different things in life. (2) He looks for adventures and likes to take risks. He wants to have an exciting life.

Security: (1) It is important to him to live in secure surroundings. He avoids anything that might endanger his safety. (2) It is very important to him that his country be safe from threats from within and without. He is concerned that social order be protected.

Tradition: (1) He thinks it's important not to ask for more than what you have. He believes that people should be satisfied with what they have.

(2) Religious belief is important to him. He tries hard to do what his religion requires.

All items were first adjusted for each respondent's mean score on all 21 items, as recommended by Schwartz (2003). The four value scales were then calculated as the sums of their two items.

When comparing human values across Europe, we adjusted the four value scales, based on the demographic composition of the regions. We estimated linear regression models that controlled for age (three linear slopes, separated at 35 and 55 years), gender, and the three Ess rounds, plus 159 random effects for the regions. All four scales showed sufficient geographical variation to warrant further analysis of the four value scales as indicated by the 159 unexplained random effects. Variance statistics revealed unexplained (by age and gender) variations that ranged from $3.2 \%$ (for stimulation) to $10.9 \%$ (for security; rho statistics in Table 1). 
TABLE 1 Unexplained variation from multilevel (random intercept) models of the four value scales, after adjusting for demographic characteristics of the respondents in ESs, rounds 5-7

\begin{tabular}{lllll} 
Statistical parameter & Self-direction & Stimulation & Security & Tradition \\
\hline SD across Regions & $0.33^{2}$ & 0.334 & 0.535 & 0.235 \\
SD across Individuals & 1.483 & 1.826 & 1.528 & 1.142 \\
Rho & 0.048 & 0.032 & 0.109 & 0.041 \\
\hline
\end{tabular}

ESs: European Social Survey; SD: standard deviation.

\subsection{Descriptive Statistics}

As many as $79 \%$ of the households had fast internet (broadband) connections in 2014, and 77\% of respondents (aged 16-74) used the internet daily (Table 2). However, internet access and internet use varied among the 159 regions, after controlling for demographic composition. In some regions, the internet was accessible to less than half the population, and other regions showed nearly full coverage. The variation in internet use $(\mathrm{SD}=12$ percentage points; hereafter $\mathrm{pp}$ ) was higher than the variation in broadband connections ( $\mathrm{SD}=9 \mathrm{pp})$.

European regions also differed in economic development and population density. Broadband costs money; and the costs are even higher in sparsely populated regions than in densely populated regions. Therefore, in the regional-level analysis, we adjusted the statistical models for two structural characteristics of the regions: the price-adjusted gross domestic product (GDP) and population density. Both variables were downloaded from Eurostat for 2014.

The four value scales were correlated internally (Table 3 ) and these correlations led to multicollinearity in regression models. Consequently, the statistical analysis proceeded stepwise: first, we investigated correlations between single value scales and internet access or internet use as the outcome (bivariate analysis); then we added regional wealth (GDP) and population density as structural variables; next, we included all four value scales in the same model. Finally, we identified which combinations of values best predicted the two outcome variables.

We also performed a longitudinal analysis to test the hypothesis of reverse causation. In this analysis, value scales were the outcome variables, and internet access and internet use were the explanatory variables. The results indicated how much the relevant value scales (from the main analysis) changed over 
TABLE 2 Descriptive statistics of the cross-sectional data (collected in 2010-14 for value scales and collected in 2014 for other variables) at the level of 159 European (NUTS2) regions

\begin{tabular}{lcccc}
\hline Variable & Mean & SD & Minimum & Maximum \\
\hline Internet access & 79.0 & 9.1 & 47.4 & 98.4 \\
Internet use & 76.6 & 11.8 & 47.2 & 96.4 \\
Self-direction & 0.03 & 0.29 & -1.3 & 0.5 \\
Stimulation & -0.03 & 0.29 & -1.0 & 0.7 \\
Security & 0.08 & 0.40 & -1.2 & 1.0 \\
Tradition & -0.01 & 0.25 & -0.5 & 0.7 \\
GDP & 1.04 & 0.34 & 0.3 & 2.1 \\
Population density & 0.43 & 0.91 & 0.0 & 7.4 \\
\hline
\end{tabular}

NUTS2: Classification of Territorial Units for Statistics; GDP: gross domestic product (a measure of regional wealth).

TABLE 3 Correlations between the variables included in the cross-sectional analysis of 159 European regions

Variable Access Use Self-dir. Stim. Security Trad. GDP P.dens.

$\begin{array}{lrrrrrrrrr}\text { Internet access } & 1.00 & & & & & & & \\ \text { Internet use } & 0.90 & 1.00 & & & & & & \\ \text { Self-direction } & 0.75 & 0.69 & 1.00 & & & & & \\ \text { Stimulation } & 0.39 & 0.49 & 0.22 & 1.00 & & & & \\ \text { Security } & -0.60 & -0.63 & -0.60 & -0.58 & 1.00 & & & \\ \text { Tradition } & -0.49 & -0.63 & -0.64 & -0.44 & 0.56 & 1.00 & & \\ \text { GDP } & 0.65 & 0.58 & 0.57 & 0.29 & -0.58 & -0.46 & 1.00 & \\ \text { Pop. density } & 0.26 & 0.22 & 0.10 & 0.22 & -0.16 & -0.14 & 0.50 & 1.00\end{array}$

the 10-year period (2004-14) between rounds 2 and 7 of the Ess, at the level of 25 countries (because NUTS2 regional classifications were only available from round 5 of the ESs). In this analysis, the relevant value scales were adjusted for the demographic (age and gender) composition of the countries, similar to the adjustments applied to the regions in the cross-sectional analysis (above).

All statistical analyses were performed with population weights using the regional populations for 2014 in the cross-sectional analyses, and for 2008 in 
the longitudinal analysis. Test statistics were adjusted for the fact that regions were more similar within than across countries, using the cluster option in Stata (Cameron and Miller 2015).

\section{$4 \quad$ Results}

\subsection{Broadband Connections}

European regions that held openness-to-change values (measured as selfdirection and stimulation) had more broadband connections at home than regions that did not hold the two openness-to-change values (Table 4). The self-direction effect was stronger and more consistent than the stimulation effect. Compared to the stimulation effect, the self-direction effect had a steeper regression slope and a narrower standard error. Moreover, it remained strong and significant after adjusting for the regions' structural characteristics (i.e., GDP and population density).

All human values were additive indices of two items each that ranged from 1 (not at all like me) to 6 (very much like me) in the questionnaire. A unit-change in self-direction (and other value indices) indicated that the mean value of

TABLE 4 Broadband connections at home in European regions as results of four value scales, estimated in separate models for each value scale (model 1-3) and jointly (model 4), regression slopes (indicating percentage point differences) with standard errors in parentheses

\begin{tabular}{|c|c|c|c|c|}
\hline \multirow[t]{2}{*}{ Human value } & 1 & 2 & 3 & 4 \\
\hline & Bivariate & + GDP & + Pop. dens. & Multivar. \\
\hline Self-direction & $\begin{array}{l}23.0^{* * *} \\
(1.9)\end{array}$ & $\begin{array}{l}17.6 \text { *** } \\
(2.3)\end{array}$ & $\begin{array}{l}18.1^{* * *} \\
(2.8)\end{array}$ & $\begin{array}{l}19.0^{* * *} \\
(4.5)\end{array}$ \\
\hline Stimulation & $\begin{array}{l}12.4 \\
(6.6)\end{array}$ & $\begin{array}{c}7 \cdot 1 \\
(5 \cdot 5)\end{array}$ & $\begin{array}{c}7.4 \\
(5.6)\end{array}$ & $\begin{array}{c}7 \cdot 5 \\
(5 \cdot 0)\end{array}$ \\
\hline Security & $\begin{array}{c}-13.5^{* * *} \\
(2.2)\end{array}$ & $\begin{array}{l}-7 \cdot 5^{* * *} \\
(2.0)\end{array}$ & $\begin{array}{l}-7 \cdot 4^{* * *} \\
(2.0)\end{array}$ & $\begin{array}{c}0.1 \\
(3.5)\end{array}$ \\
\hline Tradition & $\begin{array}{c}-17.7^{*} \\
(6.7)\end{array}$ & $\begin{array}{l}-8.8 \\
(5 \cdot 5)\end{array}$ & $\begin{array}{l}-8.6 \\
(5 \cdot 5)\end{array}$ & $\begin{array}{c}5 \cdot 1 \\
(4.8)\end{array}$ \\
\hline
\end{tabular}

\footnotetext{
${ }^{*} \mathrm{p}<0.05 ;{ }^{* *} \mathrm{p}<0.01 ;{ }^{* * *} \mathrm{p}<0.001$ (two-sided tests); GDP: gross domestic product (i.e., regional wealth); Pop. dens.: population density; Multivar: multivariable regression, including all human values and other preceding variables.
} 
the two items in the index changed by one half of a unit (because each index was the summary of two items). A unit-change in self-direction corresponded to 3.4 standard deviations $(1 / 0.29)$ of the population-weighted standard deviation variation among the European regions (0.29). This unit-change in the self-direction value was associated with a 23 -pp increase in broadband connections at the level of European regions (Table 4, Model 1).

Broadband connections into homes were also correlated with the region's wealth (GDP, Table 4, Model 2), but not its population density (Model 3; full regression results from Model 4 are presented in the appendix, Table A1.) When we controlled for regional wealth (Model 2), the self-direction effect dropped from 23 to $17.6 \mathrm{pp}$. Thus, regional wealth explained $24 \%$ of the self-direction effect, and $76 \%$ remained unexplained when we controlled for regional wealth (comparing Models 1 and 2). As much as $78 \%$ of the self-direction effect remained unexplained when we controlled for both structural characteristics of the regions (comparing Models 1 and 3).

European regions that held conservation values (measured as security and tradition) had fewer broadband connections at home than regions that did not hold the two conservation values (Table 4). The security effect was more consistent across regions than the tradition effect, because its standard error was smaller. Furthermore, security values varied more among European regions (weighted $\mathrm{SD}=0.40$ ) than tradition values $(\mathrm{SD}=0.25)$. The security effect also remained strong and significant when we controlled for the structural characteristics of the regions (Models 2 and 3 ).

A unit increase in the security index (corresponding to one half of a unit increase in both items) was associated with a 13.5-pp decrease in broadband connections at home when we compared European regions. This estimate dropped by $45 \%$ to $7.4 \mathrm{pp}$ when we controlled for the structural characteristics of the regions. This drop was largely explained by regional wealth (GDP). Hence, $55 \%$ of the security effect remained unexplained, when we controlled for the two structural characteristics of this analysis (-7.4/-13.5).

The analyses in Models 1-3 in Table 4 did not control for other human values. Openness-to-change and conservation values were also correlated internally (Table 3), and their correlation with internet access and use was also correlated, a phenomenon known as collinearity. When several openness-to-change and conservation values are entered in an additive (regression) model, they must share explanatory power. Model 4 was the same as Model 3 (controlled for regional wealth and population density), except that it included all four value scales. The security effect was not consistent, when we controlled for other human values and structural characteristics of the regions. Thus, the security effect was explained partially by structural characteristics and partially 
by self-direction values. Hence, the results indicated that self-direction was the most consistent human value predictor of the diffusion of internet connections in Europe.

\subsection{Internet Use}

Internet use (Table 5) was even more strongly correlated with general human values than internet connections (Table 4). A unit increase in self-direction values at the level of European regions was associated with a 28-pp increase in daily internet activity at home (Model 1). This statistical association between human values and internet activities was partially explained by the fact that internet activities at home were also correlated with regional wealth (Model 2), but population density did not contribute to the association (Model 3). Nevertheless, regional wealth could only explain $23 \%$ of the statistical association between self-direction values and internet activities; thus, $77 \%$ remained unexplained after the model was controlled for regional wealth (21.6/28). Stimulation values were also correlated with internet activity, but this effect was smaller and less consistent between regions, compared to selfdirection, indicated by the larger standard errors.

TABLE 5 Daily internet use at home in European regions as results of four value scales, estimated in separate models for each value scale (model 1-3) and jointly (model 4-7), regression slopes (indicating percentage point differences) with standard errors in parentheses

\begin{tabular}{|c|c|c|c|c|c|c|c|}
\hline \multirow[t]{2}{*}{ Human value } & $\mathbf{1}$ & 2 & 3 & 4 & 5 & 6 & 7 \\
\hline & Bivariate & + GDP & $\begin{array}{l}\text { + Pop. } \\
\text { dens. }\end{array}$ & \multicolumn{4}{|c|}{$\begin{array}{l}\text { Multivariable models including } \\
\text { GDP \& population density }\end{array}$} \\
\hline Self-direction & $\begin{array}{l}28.0^{* * *} \\
(4.0)\end{array}$ & $\begin{array}{l}21.6^{* * *} \\
(3.7)\end{array}$ & $\begin{array}{l}22.1^{* * *} \\
(4.2)\end{array}$ & $\begin{array}{l}16.4^{* * *} \\
(4 \cdot 7)\end{array}$ & $\begin{array}{l}20.6^{* * *} \\
(3.0)\end{array}$ & $\begin{array}{l}17.8^{* * *} \\
(4.4)\end{array}$ & $\begin{array}{l}15.6^{* * *} \\
(3 \cdot 7)\end{array}$ \\
\hline Stimulation & $\begin{array}{l}19.7^{* *} \\
(6.9)\end{array}$ & $\begin{array}{l}14.1^{*} \\
(5 \cdot 7)\end{array}$ & $\begin{array}{l}14 \cdot 5^{*} \\
(5 \cdot 7)\end{array}$ & $\begin{array}{c}9 \cdot 7 \\
(6.4)\end{array}$ & $\begin{array}{l}12.8^{* * *} \\
(3.4)\end{array}$ & & \\
\hline Security & $\begin{array}{l}-18.5^{* * *} \\
(2.4)\end{array}$ & $\begin{array}{c}-13.1{ }^{* * *} \\
(1.7)\end{array}$ & $\begin{array}{c}-13.0^{* * *} \\
(1.8)\end{array}$ & $\begin{array}{l}-2.0 \\
(4.6)\end{array}$ & & $\begin{array}{l}-8.2^{* * *} \\
(2.1)\end{array}$ & \\
\hline Tradition & $\begin{array}{l}-29.3^{* * *} \\
(6.7)\end{array}$ & $\begin{array}{l}-21.5^{* * *} \\
(5 \cdot 7)\end{array}$ & $\begin{array}{c}-21.3^{* * *} \\
(5.8)\end{array}$ & $\begin{array}{l}-7.1 \\
(5.8)\end{array}$ & & & $\begin{array}{r}-13.2^{*} \\
(5.2)\end{array}$ \\
\hline
\end{tabular}

${ }^{*} \mathrm{p}<0.05 ;{ }^{* *} \mathrm{p}<0.01 ;{ }^{* * *} \mathrm{p}<0.001$ (two-sided tests); GDP: gross domestic product (i.e., regional wealth); Pop. dens.: population density. 
The two conservation values, security and tradition, were negatively correlated with internet activities at home. Both conservation values remained significantly correlated with internet use, even after we controlled for the regions' wealth and population density (Model 3), where a unit increase in the security value index was associated with a 13-pp decrease in daily internet activity at home. In a similar analysis, a unit increase in the tradition index was associated with a 21.3-pp decrease in daily internet activity.

When we controlled for all four value scales (Model 4 in Table 5), only selfdirection values remained statistically significant. This result was the same as the result found in the analysis of internet connections (Table 4). However, when we only specified two human values, we found that, after controlling for self-direction values, the three the other human values (stimulation, security, and tradition) remained statically significant (Models 5-7 in Table 5). Still, selfdirection was by far the most consistent predictor of internet use at the level of European regions. Hence, the final analysis provided a choice of three different models to explain the relationship between human values and internet use. To resolve this situation, we investigated which of the three models (5-7) fit the data best. R-squared statistics for Model 5 were higher $(62 \%)$ than for Models 6 and 7 ( $57 \%$ and $58 \%$ respectively). Hence, the results indicated that a combination of self-direction and stimulation values best predicted the diffusion of internet use in Europe. We should keep in mind, however, that conservation values could also predict internet use, even though the data fits were slightly worse than the fits observed for openness-to-change values.

\subsection{Reverse Causation}

Our investigation of the ability of four general human values to predict the diffusion of the internet by 2014 among 159 European regions indicated that openness-to-change values were the best predictors of internet diffusion into European homes. However, it was also possible that openness-to-change values might have changed as consequence of the internet diffusion in Europe. This issue of reverse causation could be investigated empirically in panel data, because the csis data were available for a reasonable number of countries, starting in 2004, and because the Ess provided data on general human values biannually over the same period. Thus, we conducted a longitudinal analysis, at the level of countries, with biannual panel data collected from 2004 to 2014 .

For the dependent variables, we used Ess data on general human values for every available round (2-7) from 2004 to 2014. For the explanatory variables, we used the csis data on internet connections and internet use, collected during the same years (biannually from 2004 to 2014). These data were not corrected for demographic characteristics. This analysis included 122 combinations 
of countries and years. Switzerland dropped out of this longitudinal analysis, because we only had data on internet use in Switzerland starting in 2014. Moreover, data for one or more years were missing for eight other countries.

Openness-to-change values increased slightly over the 10-year period we investigated. We reasoned that this change would probably best be estimated in a panel data model, due to the missing values for some combinations of countries and years. We found that self-direction increased by $0.08(\mathrm{p}<5 \%)$ and stimulation increased by 0.03 (non-significant) over the 10-year study period, with a linear period effect and fixed effects for the 25 countries analyzed.

Table 6 presents longitudinal estimates for the relationships between the two openness-to-change values (dependent variables) and the diffusion of broadband connections and internet use (explanatory variables), with fixed effects and population weights for the countries. It shows the estimates from eight regression models, with all combinations of the two dependent variables and two explanatory variables, either with (models labeled 1 ) or without (models labeled 2) adjustments for period effects (wave dummies). We expressed the outcome variable as a proportion (from o to 1 ) rather than percentage (from 0 to $100 \%$ ). A panel model with no period effects merely showed the amount of change in two variables (human values and internet connections/use) during the study period. When we added period effects, the model indicated whether

TABLE 6 Longitudinal analysis of the relationships between openness-to-change values (self-direction and stimulation) and the proportions of individuals with broadband connections or daily internet use, at the level of 25 countries $(\mathrm{N}=122)$; regression slopes with standard errors in parentheses

\begin{tabular}{lcccc} 
Parameter & \multicolumn{2}{l}{ Self-direction } & \multicolumn{2}{l}{ Stimulation } \\
Model & $\mathbf{1}$ & $\mathbf{2}$ & $\mathbf{1}$ & $\mathbf{2}$ \\
\hline Broadband connection & $0.15^{* *}$ & -0.26 & 0.06 & 0.05 \\
SE & $(0.04)$ & $(0.22)$ & $(0.04)$ & $(0.20)$ \\
Adjusted for period effects & No & Yes & No & Yes \\
Model & & & & \\
Internet use & $\mathbf{1}$ & $\mathbf{2}$ & $\mathbf{1}$ & $\mathbf{2}$ \\
SE & $0.35^{* *}$ & -0.21 & 0.15 & 0.15 \\
Adjusted for period effects & $(0.11)$ & $(0.25)$ & $(0.10)$ & $(0.27)$ \\
& No & Yes & No & Yes
\end{tabular}

** $\mathrm{p}<0.01$ (two-sided tests). 
countries that experienced a change greater than the mean change in an explanatory variable (internet connections or internet use) also experienced a change greater than the mean change in the outcome variable (openness-tochange values).

Self-direction values increased during the same period as broadband connections and internet use expanded (Model 1). However, when we controlled for period effects (Model 2), the data showed no tendency toward any additional change in self-direction values for the countries with the strongest increases in broadband connections and internet use. Similarly, we found no correlations between the changes in stimulation values and the increases in broadband connections and internet use. Hence, the hypothesis of reverse causation - that the diffusion of the internet affected openness-to-change values - could be rejected.

\section{5}

\section{Discussion}

The relationship between technological/economic change and cultural change, or between material structures and human values, has been the subject of long-standing controversy, certainly within sociology. Materialists, such as Karl Marx, have argued that economic and technological changes tend to precede cultural values; conversely, idealists, such as Max Weber, have argued that cultural values may also affect economic development. More recent theorists, such as Ronald Inglehart (1997), have argued that cultural changes in human values reflect changes in the economic environment, but they take place with a generational time lag and have explanatory power of their own.

Currently, it is possible to investigate some of these theoretical arguments and controversies empirically, because recent technologies (e.g., the internet) have been implemented over relatively few years, and because value instruments allow us to investigate cultural values and how they change over several years. The present study began to address these issues by investigating data on the diffusion of the internet in combination with data on general human values. The results indicated that openness-to-change values predicted the internet diffusion into homes. Furthermore, internet diffusion could not predict a similar change in openness-to-change values.

Previous studies have identified correlations between Hofstede-type values and the diffusion of the internet at the level of countries (Bagchi et al. 2004; Erumban and De Jong 2006; Takieddine and Sun 2015). However, most of those correlations either disappeared, when the model was controlled for national wealth (Bagchi et al. 2004), or remained significant only when national wealth 
was classified into rich and poor countries (Erumban and De Jong 2006). Hence, those studies provided little empirical support for the hypothesis that human values affected the diffusion of the internet beyond economic factors. In contrast, the current study indicated that some human values were stronger than regional wealth as predictors of the diffusion of the internet (Appenix Table A1). The current study was different, because we studied a lower geographical level (regions instead of countries), we used different value scales (Schwartz instead of Hofstede scales), and we controlled for the regions' demographic compositions, by implementing standardization and multilevel methods.

Comparative research has always struggled with a small number of countries. However, when the number of countries is expanded, the units (countries) become more heterogeneous, which could make it difficult to estimate true relationships between variables. Aggregates lower than nations (i.e., regions) are promising alternatives, because they provide more observations among units that are not excessively dissimilar.

Like previous studies, we faced situations of multicollinearity. In previous studies, this occurred when Hofstede-type values were added to a model, together with national wealth. In the present study, it occurred when several general human values were added into the same statistical model for analyzing internet connections and use in different regions. When we investigated single human values, we found that all four human value scales could partly explain the diffusion of internet use beyond the regions' economic wealth. However, many of these correlations became insignificant in models that included more than one human value at a time. In these situations, it can be difficult to choose among different empirical models that include two or more human values. Nevertheless, our major finding was that openness-to-change values, in general, and self-direction values, in particular, fit the data on internet connections and internet use better than conservation values.

Openness-to-change values refer to how we relate to any social change (Devos et al. 2007). The sub-dimension, self-direction, is the preference to make one's own decisions, instead of being told what to do by others. In other words, self-directed people value individualization. The sub-dimension, stimulation, is the motivation to seek excitement and novel experiences.

The finding that cultural enthusiasm for any social change (i.e., opennessto-change values at the level of regions) was associated with the diffusion of internet use might be self-evident, because the internet represents a major societal change, including a change in domestic life. Internet access provides people with both individual choices and excitement (both aspects of opennessto-change values). The correlation between enthusiasm for individual choice (self-direction) and the diffusion of internet connections is more fascinating, 
because enthusiasm for individual choice (individualism) represents only one aspect of a changing world.

The longitudinal analysis indicated that self-direction values were not affected by the expansion of the internet. Taken together, these findings indicate that the expansion of the broadband connections was facilitated by dominant values in regional populations that reflected how much they valued individualism. There is also the possibility that policy makers and commercial actors held these same values, or they were at least aware of these values in their target populations. Previous research has shown that telecommunication policies (i.e., deregulation) might also have predicted the diffusion of the internet (Dauvin and Grzybowski 2014; Guillen and Suarez 2005). One implication of the current study (and the arguments above) is that even telecommunication policies might be an outcome of the general human values held by the populations of various countries and regions. This issue should be subject of future research.

\subsection{Limitations}

This study had limitations. We could only investigate the diffusion of the internet before 2014 at the level of countries, and only back to 2004. By 2014, nearly all European regions had achieved broadband connections in half of all households. By 2004, broadband connections had been achieved in more than 30\% of households in some countries. Hence, the present study identified the role of human values at somewhat mature stages of internet diffusion.

There are also some uncertainties related to comparing human values across countries or language groups. Previous studies have compared factor loadings (metric invariance), sometimes in combination with intercepts (scale invariance), in the value scales of the Ess (e.g., Cieciuch et al. 2018). The results indicated that the higher-order openness-to-change value was reasonably comparable across countries, when hedonism was excluded (as in the current study), but the higher-order conservation value (security and tradition) was less comparable across countries (Cieciuch et al. 2018).

\subsection{Conclusions}

This study has identified strong empirical relationships between openness-tochange values, and the self-direction value particularly, and the diffusion of the internet at the level of European regions. These relationships remained strong and significant also when the statistical models were controlled for regional wealth. 


\section{Funding}

The author received no financial support for the research, authorship, and/or publication of this article.

\section{References}

Bagchi. K., Hart, P. and Peterson, M.F. 2004. "National Culture and Information Technology Product Adoption." Journal of Global Information Technology Management 7(4): 29-46.

Bagchi, K.K., Godwin, J.U., Peeter, J.K. and Khendum, C. 2015. "Internet Use and Human Values: Analyses of Developing and Developed Countries." Computers in Human Behavior 50: 76-90.

Bardi, A., Lee, J.A., Hofmann-Towfigh, N. and Soutar, G.N. 2009. "The Structure of Intraindividual Value Change." Journal of Personality and Social Psychology 97(5): 913-929.

Bobkowski, P. and Smith, J. 2013. "Social Media Divide: Characteristics of Emerging Adults Who Do Not Use Social Network Websites." Media, Culture \& Society 35(6): 771-781.

Boltanski, L. and Laurent, T. 1999. "The Sociology of Critical Capacity." European Journal of Social Theory 2(3): 359-377.

Cameron, A.C. and Miller, D.L. 2015 “A Practitioner's Guide to Cluster-Robust Inference.” Journal of Human Resources 50(2): 317-372.

Cieciuch, J., Davidov, E., Algesheimer, R. and Schmidt, P. 2018 “Testing for Approximate Measurement Invariance of Human Values in the European Social Survey." Sociological Methods \& Research 47(4): 665-686.

Crenshaw, E.M. and Robison, K.K. 2006. "Globalization and the Digital Divide: The Roles of Structural Conduciveness and Global Connection in Internet Diffusion." Social Science Quarterly 87(1): 190-207.

Daniel, E., Fortuna, K., Thrun, S.K. and Knafo, A. 2013. "Early Adolescents' Value Development at War Time." Journal of Adolescence 36(4): 651-655.

Dauvin, M. and Grzybowski, L. 2014. "Estimating Broadband Diffusion in the EU Using NUTS1 Regional Data." Telecommunication Policies 38(1): 96-104.

Devos, G., Buelens, M. and Bouckenooghe, D. 2007. "Contribution of Content, Context, and Process to Understanding Openness to Organizational Change: Two Experimental Simulation Studies." The Journal of Social Psychology 147(6): 607-630.

DiMaggio, P. and Garip, F. 2011. "How Network Externalities Can Exacerbate Intergroup Inequality." American Journal of Sociology 116(6): 1887-1933. 
Erumban, A.A. and de Jong, S.B. 2006. "Cross-Country Differences in ICT Adoption: A Consequence of Culture?" Journal of World Business 41(4): 302-314.

Fischer, C.S. 1992. America Calling: A Social History of the Telephone to 1940. Berkeley and Los Angeles: University of California Press.

Guillen, M.F. and Suarez, S.L. 2005. "Explaining the Global Digital Divide: Economic, Political and Sociological Drivers of Cross-National Internet Use." Social Forces 84(2): 681-708.

Hadler, M. 2006. "Intentions to Migrate Within the European Union: A Challenge for Simple Economic Macro-Level Explanations.” European Societies 8(1): 111-140.

Hargittai, E. and Hsieh, Y.P. 2013. “Digital Inequality." Pp. 129-150 in The Oxford Handbook of Internet Studies, edited by W.H. Dutton, Oxford: Oxford University Press.

Helsper, E.J. and Reisdorf, B.V. 2017. "The Emergence of a "Digital Underclass" in Great Britain and Sweden: Changing Reasons for Digital Exclusion." New Media \& Society 19(8): 1253-1270.

Hofstede, G. 1980. Culture's Consequences: International Differences in Work-Related Values. Beverly Hills: Sage.

Hofstede, G. 1998. Masculinity and Femininity: The Taboo Dimension of National Cultures. Thousand Oaks: Sage.

Hofstede, G. 2001. Culture's Consequences: Comparing Values, Behaviours, Institutions and Organizations Across Nations. Thousand Oaks: Sage.

Homer, P.M. and Kahle, L.R. 1988. "A Structural Equation Test of the Value-AttitudeBehavior Hierarchy." Journal of Personality and Social Psychology 54(4): 638-646.

Inglehart, R. 1990. Culture Shift in Advanced Industrial Society. Princeton: Princeton University Press.

Inglehart, R. 1997. Modernization and Postmodernization: Cultural, Economic, and Political Change in 43 Societies. Princeton: Princeton University Press.

Korupp, S.E. and Szydlik, M. 2005. "Causes and Trends of the Digital Divide." European Sociological Review 21(4): 409-422.

Lesthaeghe, R. 2014. "The Second Demographic Transition: A Concise Overview of Its Development." Proceedings of the National Academy of Sciences of the United States of America 111(51): 18112-18115.

Lizardo, O. 2017. "Improving Cultural Analysis: Considering Personal Culture in Its Declarative and Nondeclarative Modes." American Sociological Review 82(1): 88-115.

Merton, R.K. 1968. Social Theory and Social Structure. New York: Free Press.

Pick, J.B., Sarkar, A. and Johnsen, J. 2015. "United States Digital Divide: State Level Analysis of Spatial Clustering and Multivariate Determinants of ICT Utilization." Socio-Economic Planning Sciences 49(1): 16-32.

Piurko, Y., Schwartz, S.H. and Davidov, E. 2011. "Basic Personal Values and the Meaning of Left-Right Political Orientations in 20 Countries." Political Psychology 32(4): 537-561. 
Rokeach, M. 1973. The Nature of Human Values. New York: Free Press.

Schwartz, S.H. 1992. "The Universal Content and Structure of Values: Theoretical Advances and Empirical Tests in 20 Countries." Advances in Experimental Social Psychology 25:1-65.

Schwartz, S.H. 2003. A Proposal for Measuring Value Orientations Across Nations. Available at: http://ess.nsd.uib.no/ess.

Schwartz, S.H. 2012. "An Overview of the Schwartz Theory of Basic Values." Online Readings in Psychology and Culture 2(1): https://doi.org/10.9707/2307-0919.1116.

Swidler, W. 1986. "Culture in Action: Symbols and Strategies." American Sociological Review 51(2): 273-286.

Takieddine, S. and Sun, J. 2015. "Internet Banking Diffusion: A Country-Level Analysis." Electronic Commerce Research and Applications 14(5):361-371.

Vaisey, S. 2009. "Motivation and Justification: A Dual Process Model of Culture in Action." American Journal of Sociology 114(6): 1675-1715.

Van Deursen, A.J.A.M. and Van Dijk, J.A.G.M. 2011. "Internet Skills and the Digital Divide." New Media \& Society 13(6): 893-911.

Vecchione, M., Schwartz, S.H., Alessandri, G., Doring, A.K., Castellani, V. and Caprara, M.G. 2016. "Stability and Change of Basic Personal Values in Early Adulthood: An 8-Year Longitudinal Study." Journal of Research in Personality 63: 111-122.

Vicente, M.R. and López, A.J. 2011. "Assessing the Regional Digital Divide across the European Union-27." Telecommunications Policy 35(3): 220-237.

Weber, M. 1934. Die Protestantische Ethik und der Geist des Kapitalismus. Tübingen: Mohr.

Wu, L.F., Cai, Y. and Liu, D. 2011. "Online Shopping among Chinese Consumers: An Exploratory Investigation of Demographics and Value Orientation." International Journal of Consumer Studies 35(4): 458-469.

Zhao, S. 2006. "The Internet and the Transformation of the Reality of Everyday Life: Toward a New Analytic Stance in Sociology." Sociological Inquiry 76(4): 458-474. 


\section{Appendix}

TABLE A1 Full regression results from Tables 4 and 5

\begin{tabular}{lcccc}
\hline Test variable & \multicolumn{2}{c}{ Broadband connections } & Internet use & \\
& Coefficient & SE & Coefficient & SE \\
\hline Self-direction & $18.1^{* * *}$ & $(2.8)$ & $20.6^{* * *}$ & $(3.0)$ \\
Stimulation & & & $12.8^{* * *}$ & $(3.4)$ \\
GDP & $7 \cdot 7^{*}$ & $(3 \cdot 3)$ & 6.8 & $(3.6)$ \\
Population density & 0.6 & $(0.6)$ & 0.1 & $(0.5)$ \\
Constant & $70.2^{* * *}$ & $(4 \cdot 3)$ & $69.3^{* * *}$ & $(5.2)$ \\
\hline
\end{tabular}

${ }^{*} \mathrm{p}<0.05 ;{ }^{* *} \mathrm{p}<0.01$; *** $\mathrm{p}<0.001$ (two-sided tests); SE: standard error; GDP: gross domestic product (a measure of regional wealth). 